\title{
SDF-1/54-DCN: A Novel Recombinant Chimera with Dual Inhibitory Effects on Proliferation and Chemotaxis of Tumor Cells
}

\author{
Weifeng MA, ${ }^{a}$ Shaoxi CAI,${ }^{a}$ Jun Du, ${ }^{b}$ Yi TAN, ${ }^{a}$ Hongyuan Chen, ${ }^{a}$ Zhigang Guo, ${ }^{a}$ Houwen Hu, ${ }^{c}$ \\ Rui FANG, ${ }^{c}$ and Shaohui CAI ${ }^{*, c}$ \\ ${ }^{a}$ College of Bioengineering, Key Laboratory for Biomechanics \& Tissue Engineering of the State Ministry of Education, \\ Chongqing University; Chongqing 400044, China: ${ }^{b}$ School of Pharmaceutical Sciences, Sun Yat-sen University; \\ Guangzhou 510080, China: and ${ }^{c}$ Department of Clinical Pharmacology, College of Pharmacy, Jinan University; \\ Guangzhou 510632, China. Received November 29, 2007; accepted March 19, 2008; published online March 24, 2008
}

Several studies have shown that the interaction of CXC chemokine receptor 4 (CXCR4) with its ligand, stromal cell-derived factor-1 $\alpha$ (SDF-1 $\alpha)$ is closely involved in the directional migration of some tumors toward specific organs, which provides a new pathway against cancer metastasis. We previously developed an $\alpha$-helix-defective mutant of SDF-1 $\alpha$, SDF-1/54 that displays obvious antagonistic activity to CXCR4. But it is necessary to ensure the targeting of SDF-1/54 to tumors in vivo since many normal tissues also express CXCR4. It is known that most tumor cells highly express epidermal growth factor receptor (EGFR). Meanwhile, decorin (DCN), a specific antagonist of EGFR, can target the tumor cells enriched in EGFR and cause a significant downregulation of EGFR. Hereby, we further generated a fusion construct of SDF-1/54 and DCN to expect to enhance the targeting of SDF-1/54 to tumors by dual blocking effects on CXCR4 and EGFR. This study focused on expression of recombinant chimera SDF-1/54-DCN in Escherichia coli, purification and bioactivity to inhibit the physiological functions mediated by CXCR4 and EGFR respectively in various tumor cell lines in vitro. Results indicated that SDF-1/54-DCN could inhibit both chemotaxis and proliferation of the tumor cells we used, which may be attributed to its blocking to CXCR4 and EGFR. These findings suggest that this strategy to link SDF-1/54 with DCN may be a promising approach to increase the targeting of SDF-1/54 to the tumors coexpressing CXCR4 and EGFR.

Key words stromal cell-derived factor- $1 \alpha$; decorin; chimera; chemotaxis; proliferation

CXC chemokine receptor 4 (CXCR4) and its ligand, stromal cell-derived factor- $1 \alpha(\mathrm{SDF}-1 \alpha)$ are believed to be involved in the directional migration of many tumors toward specific organs. ${ }^{1-3)}$ Muller A., et al. reported that metastasis of breast cancer cells is the result of several sequential steps. It represents a highly organized, non-random, and organ-selective process wherein signaling via CXCR4 or CC chemokine receptor 7 (CCR7) mediates actin polymerization and pseudopodia formation and subsequently induces chemotactic and invasive responses. ${ }^{4)}$ This metastatic mode was also identified in a variety of cancers such as oral squamous carcinoma, ${ }^{5)}$ small cell lung cancer, ${ }^{6}$ and prostate cancer, ${ }^{7)}$ and it may help to establish a new target and strategy for prevention and treatment of cancer metastasis. Based on this concept, we previously developed SDF-1/54, an $\alpha$-helixdefective mutant of SDF- $1 \alpha$, and examined its antagonistic activity in CXCR4-mediated signaling and chemotaxis of tumor cells. ${ }^{8,9)}$ However, CXCR4 also widely expresses on normal tissues and cells, including brain, heart, kidney, liver, lung, spleen and immunologic cells, and plays vital roles in central nervous system and immunologic system development. ${ }^{10)}$ Accordingly, SDF-1/54 will unavoidably induce serial adverse reactions due to systemic administration, which indicates the significance to improve the targeting capability of SDF-1/54 to tumors in vivo.

Epidermal growth factor receptor (EGFR), a member of the $\operatorname{erb} B$ family of receptor tyrosine kinases, ${ }^{11)}$ is highly expressed in a variety of solid tumors, including breast, head and neck, non-small cell lung, and prostate cancers, etc. In some tumor tissues, EGFR is associated with an advanced tumor stage, i.e., with poor prognosis and resistance to chemo-, radio-, and hormone therapy. ${ }^{12)}$ Decorin (DCN), a small molecular glycoprotein rich in leucine, has been demonstrated as a natural specific inhibitor of EGFR. ${ }^{13-15)}$ It is known that DCN can bind to a discrete region of EGFR, which is partially overlapping but distinct from the EGFbinding domain, and inhibit the growth of a variety of transformed cells and tumor xenografts. ${ }^{16,17)}$ Recently, Seidler D. G., et al. discovered that DCN specifically targeted the tumor cells enriched in EGFR and caused a significant downregulation of EGFR and attenuation of its activity within the orthotopic squamous carcinoma xenograft, ${ }^{18}$ ) which provided a valuable idea that DCN might function as a delivery vehicle for targeting therapy to various human tumor cells where EGFR played a key pathophysiological role. Based on the above-mentioned facts, we assume that linking SDF-1/54 with DCN probably improves the targeting capability of SDF-1/54 to the tumor cells that express both CXCR4 and EGFR on their surface.

A number of studies have testified that the application of fusion proteins is a potential way to improve not only therapeutic effect but also targeting ability of each unit in chimera. Azemar M., et al. reported that the recombinant antibody toxins consisted of the EGFR/ErbB2-specific singlechain antibody ( $\mathrm{scFv}$ ) and the truncated Pseudomonas exotoxin A (ETA) inhibited the in vitro growth of human head and neck cancer cells and caused rapid tumor regression in vivo. ${ }^{19)} \mathrm{Xu}$ Y. M., et al. demonstrated that a caspase- 6 and anti-human epidermal growth factor receptor-2 (HER2) antibody chimeric molecule suppressed the growth of HER2overexpressing tumors and prolonged animal survival significantly. ${ }^{20)}$ Again, compared with combined treatment of the two individual proteins, the fusion of SDF-1/54 and DCN can ensure the consistency in the pharmacokinetic character- 
istics of these two relevant functional units, which is important for the two proteins to exert synergic effects. Therefore, it is reasonable to believe that the fusion of SDF-1/54 and DCN may be a more available strategy compared with simple mix of SDF-1/54 and DCN under systemic administration.

This study focused on expression of recombinant protein SDF-1/54-DCN in Escherichia coli, purification and bioactivities to inhibit the physiological functions mediated by CXCR4 and EGFR respectively in various tumor cell lines in vitro, which might be expected to provide evidences for the feasibility of this strategy.

\section{MATERIALS AND METHODS}

Materials E. coli $\mathrm{DH} 5 \alpha, E$. coli BL21 (DE3), and plasmid pET-30a $(+)$ were from Novagen, U.S.A. Phycoerythrin (PE)-conjugated mouse anti-human CXCR4 antibody (12G5) was from R\&D Systems. Mouse anti-human EGFR-FITC antibody was from BD Transduction Labs. Rabbit anti-His polyclone antibody (H-15, SC-803), mouse antiphosphoERK (E-4) antibody, and rabbit anti-ERK2 antibody were from Santa Cruz Biotech. Inc. Human acute lymphoblastic leukemia (MOLT-4) cells, human epithelial carcinoma (A431) cells, human breast cancer MDA-MB-361 and human embryo kidney epithelial (HEK 293) cell lines were from the China Center for Type Culture Collection.

Construction of Recombinant Plasmid SDF-1/54DCN/pET-30a(+) SDF-1/54 was amplified by polymerase chain reaction $(\mathrm{PCR})$ from $\mathrm{SDF}-1 / \mathrm{pET}-30 \mathrm{a}(+)$ with primers 5'-GGGGTACCGACGACGACGACAAGAAGCCCGTCAGCCTGAGCTACAGAT-3' (forward) and 5'-CCGCTCGAGCTTCGGGTCAATGCACAC-3' (reverse). The amplified PCR products were linked to $\mathrm{pET}-30 \mathrm{a}(+)$ after digestion with $K p n I$ and $X h o I$. The coding sequence CCCAAATCTTGTGACAAAACTCACACATGCCCACCGTGCCCA of the hinge region was synthesized by Shanghai Sangon Biotech. Co., Ltd. The complementary chains were annealed to yield double strands containing XhoI and BamHI sites at both terminals, and then linked to the expression vector SDF1/54/pET-30a $(+)$.

The DCN cDNA was cloned from human bone marrow by RT-PCR with primers 5'-CGGGATCCGATGAGGCTTCTGGGATAGGCC-3' (forward) and 5'-GGAATTCTTACTTATAGTTTCCGAGTTG-3' (reverse). The amplified products of DCN were digested with BamHI/EcoRI and then linked to SDF-1/54-Hinge/pET-30a(+).

Expression and Identification of Chimera Protein The recombinant plasmid SDF-1/54-Hinge-DCN/pET-30a $(+)$ was transformed into expression bacteria $E$. coli BL21 (DE3). The positive clone was induced with $1.0 \mathrm{~mm}$ isopropyl $\beta$-D-thiogalactoside (IPTG) at a desired cell density $(\mathrm{OD}=0.6)$. The bacteria were lysed with lysozyme and sonicated after $3 \mathrm{~h}$ culture. The supernatant and inclusion body were then assessed by SDS-PAGE and then examined by Western blotting with rabbit anti-His polyclone antibody.

Purification and Renaturation of Recombinant Protein The precipitate was washed twice with RIPA buffer $(0.1 \%$ SDS, $1 \%$ TritonX-100, $1 \%$ sodium deoxycholate, $25 \mathrm{~mm}$ Tris- $\mathrm{HCl}$ [pH 7.5], $150 \mathrm{~mm} \mathrm{NaCl}$ ) and dissolved in $8 \mathrm{M}$ urea. It was then purified by nickel affinity chromatography and HPLC with a C-18 column.
The eluent was diluted to a quadruple volume with a renaturing buffer $(20 \mathrm{~mm}$ Tris- $\mathrm{HCl}[\mathrm{pH} 9.0], 0.5 \mathrm{~mm}$ oxidized glutathione, $5 \mathrm{~mm}$ reduced glutathione, $0.5 \mathrm{~mm} \mathrm{~L}$-arginine, $0.5 \mathrm{~mm}$ L-histidine, $20 \mathrm{~nm} \mathrm{CuSO}, 1 \%$ glycine, and 5\% glycerol) and dialyzed twice against renaturing buffer and PBS at $4{ }^{\circ} \mathrm{C}$ ( $6 \mathrm{~h}$ each time). Next, the solution was lyophilized and ultrafiltrated for desalination and condensation.

Chemotaxis Assay The migration of MOLT-4 cells and MDA-MB-361 cells were assessed in disposable Transwell trays (KURABO, Osaka, Japan) with 6-mm-diameter chambers and membrane pore size of $5 \mu \mathrm{m}$ (for MOLT-4 cells) and $8 \mu \mathrm{m}$ (for MDA-MB-361 cells) respectively. SDF- $1 \alpha$ or SDF-1/54-DCN was diluted in RPMI 1640 medium containing $1 \mathrm{mg} / \mathrm{ml}$ of BSA. $700 \mu \mathrm{l}$ of each diluted sample was added to the lower wells, and $200 \mu \mathrm{l}$ of the RPMI 1640 suspension in which the cell density was $1 \times 10^{7} / \mathrm{ml}$ was added to the upper wells. After incubating in humidified $5 \% \mathrm{CO}_{2}$ at $37^{\circ} \mathrm{C}$ for $2 \mathrm{~h}$, the cells that migrated to the lower wells were counted. The control was the migrating cells without any treatment. In chemotaxis inhibition assay, various concentrations of SDF-1/54-DCN was preincubated with cells at $37^{\circ} \mathrm{C}$ for $2 \mathrm{~h}$. Two-hundred microliters of preincubated cells was added to the upper wells, and $700 \mu \mathrm{l}$ of diluted SDF-1 $\alpha$ $(0.01 \mathrm{nM})$ was added to the lower wells. For chemotaxis inhibition assay in MDA-MB-361 cells, SDF-1/54 (500 nM), DCN (500 nM) alone and combined both were set as control groups. In above each experimental process, cell viability was examined by trypan blue exclusion test according to a previously described method. ${ }^{21)}$

Cell Proliferation Assay The proliferation inhibiting activity of SDF-1/54-DCN was assessed by the MTT assay. A431 cells and MDA-MB-361 cells were resuspended at a density of $5 \times 10^{4} / \mathrm{ml}$, and $200 \mu \mathrm{l}$ suspension was then cultured in each well of a 96-well plate. After cultured for $24 \mathrm{~h}$, the cells were treated with SDF-1/54-DCN at the indicated concentrations for $48 \mathrm{~h}$, and the cell proliferation was assessed by MTT assay according to procedures described previously. ${ }^{22}$ The inhibition efficiency of SDF-1/54-DCN was calculated according to the following formula: inhibition rate of sample $=(1-$ OD of sample $/ O D$ of control $) \times 100 \%$.

EGFR and CXCR4 Internalization Assay The internalization of EGFR and CXCR4 was assayed using A431 and MOLT- 4 cell lines respectively. SDF-1/54-DCN at a final concentration of $1 \mu \mathrm{M}$ was added to $400 \mu \mathrm{l}$ of cell suspension $\left(5 \times 10^{6} / \mathrm{ml}\right)$, and PBS of an equal volume was added to the cells as control. After culture for $2 \mathrm{~h}$, the cells were stained with mouse anti-human monoclonal antibody EGFR-FITC or mouse anti-human monoclonal antibody CXCR4-PE and analyzed by flow cytometry.

Immunoblot Analysis MOLT-4 cells were stimulated by SDF-1/54-DCN or SDF- $1 \alpha$ for 2 min and lysed immediately. The expression of ERK was examined by Western blotting with primary antibodies (1:1000 dilution of mouse anti-pERK [E-4] or rabbit anti-ERK2) and horseradish peroxidaseconjugated secondary antibody (1:1000 dilution in TBST).

\section{RESULTS}

Construction and Expression of SDF-1/54-DCN/pET30a(+) The design and construction of SDF-1/54-DCN is illustrated in Fig. 1. The recombinant plasmid SDF-1/54- 


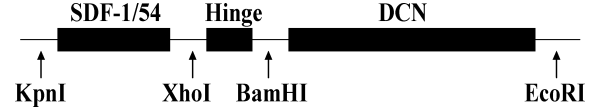

Fig. 1. Strategy for the Construction of SDF-1/54-DCN

SDF-1/54 was linked to the N-terminus of DCN by using the hinge region of human IgG1 as the linker.
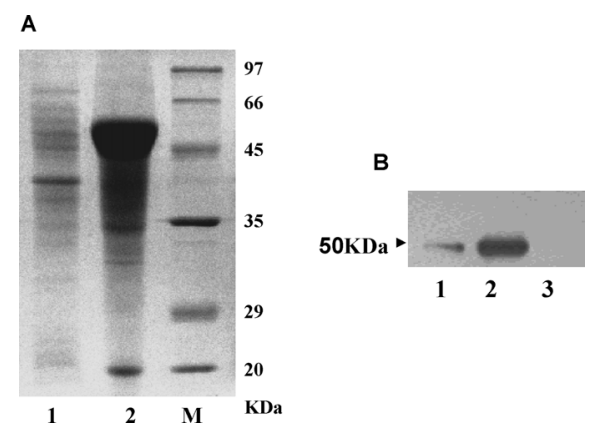

Fig. 2. Expression and Identification of SDF-1/54-DCN

(A) Expression of SDF-1/54-DCN. Lane 1: Supernatant of SDF-1/54-DCN BL21(DE3) cell lysate, lane 2:Inclusion body solution of SDF-1/54-DCN; M: Molecular weight markers. (B) Western blot assay. Expression of SDF-1/54-DCN was confirmed by Western blotting with mouse anti-His antibody. Lane 1: Supernatant of SDF1/54-DCN BL21(DE3) cell lysate; lane 2: Inclusion body solution of SDF-1/54-DCN; lane 3: pET-30a(+) BL21(DE3) cell lysate.

$\mathrm{DCN} / \mathrm{pET}-30 \mathrm{a}(+)$ was confirmed by sequencing and transformed into BL21 (DE3). The expressed products were detected by SDS-PAGE. As shown in Fig. 2A, the molecular weight of SDF-1/54-DCN was approximately $50 \mathrm{kDa}$, which is consistent with the predicted size. This recombinant chimera protein mainly existed in the inclusion body. Furthermore, we examined the expression of this chimera protein by Western blotting with mouse anti-His antibody and observed a specific immune reaction at the $50 \mathrm{kDa}$ site in the bacteria transformed with SDF-1/54-DCN/pET-30a $(+)$ but not in those transformed with pET-30a $(+)$. These results suggested the adequate expression of recombinant chimera protein SDF-1/54-DCN (Fig. 2B).

Purification and Renaturation of SDF-1/54-DCN Heterologous expression of foreign genes in $E$. coli often leads to the accumulation of expressed proteins in insoluble inclusion bodies. Inclusion bodies can be a potential initiation point for the purification of proteins since they contain almost pure protein in their inactive forms at different stages of aggregation. ${ }^{23,24)}$ However, the refolding process often raises issues such as insolubility and poor yield. In this study, we purified SDF-1/54-DCN from inclusion bodies by two steps. First, the inclusion body was thoroughly washed by RIPA buffer to remove cell debris and hybrid proteins. This chimera protein was then purified by nickel affinity chromatography. The result showed that the purity of the recombinant protein was approximately $85 \%$ under this condition (Fig. 3A). After second purification by HPLC with a C-18 column, the purity of the target protein reached more than 96\% (Fig. 3B). The purified target protein was renatured by diluting slowly, dialyzing, and ultrafiltrating stepwise. Considering that either glycine or glycerol is helpful in improving the solubility of many proteins, $1 \%$ glycine and $5 \%$ glycerol were added to the renaturing buffer to improve the renaturing efficiency and stability of SDF-1/54-DCN. The results indicated that SDF-1/54-DCN could be renatured under this
A
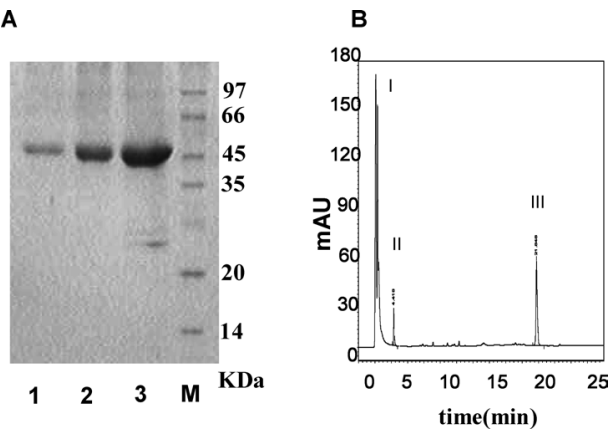

Fig. 3. Purification of SDF-1/54-DCN

(A) The results of electrophoresis of the purified SDF-1/54-DCN by ProBond ${ }^{\mathrm{TM}}$ Resin chromatography. Purity of the target protein increased to $85 \%$ after washing the inclusion body with the RIPA buffer in SDS-PAGE. Lanes 1, 2, and 3: Elution solution of SDF-1/DCN; M: Molecular weight markers. (B) HPLC chromatogram of the purified SDF-1/54-DCN. HPLC system with a C-18 column was used to purify SDF-1/54DCN. The purity of the target protein was more than $96 \%$, as determined by the percent area. I: Solvent peak; II: impurity peak; III: target peak.

condition (Figs. 4, 5).

SDF-1/54-DCN Inhibited Chemotactic Ability of SDF$1 \alpha$ in MOLT-4 Cells Given that induction of the migration of T lymphocytes through interaction with CXCR4 is an important physiological function of the native SDF-1 $\alpha,{ }^{25)}$ the chemotactic ability of SDF-1/54-DCN was assessed in MOLT-4 cells, a T lymphocyte leukemia cell line that constitutively overexpressed CXCR4. Compared with MOLT-4 cells without any treatment, SDF- $1 \alpha$ induced dose-dependent chemotaxis of MOLT 4 cells and reached the peak at a concentration of approximately $10 \mathrm{~nm}$ (Fig. 4A). Nevertheless, SDF-1/54-DCN did not show significantly chemotactic ability at the same concentration, and even though the concentration was increased to $100 \mathrm{~nm}$, its chemotactic ability still retained at lower level (Fig. 4A). Furthermore, extracellular signal-regulated kinase (ERK) signaling is known as a critical signal transduction pathway associated with SDF- $1 \alpha$ activating CXCR4. ${ }^{26}$ ) Thus we further performed experiments to detect the phosphorylation of ERK. The result showed that SDF-1/54-DCN failed to induce the activation of ERK (Fig. 4B), which implied that this new chimera protein had lost its capacity to activate intracellular signaling mediated by CXCR4. Of course, there was another possible explanation that SDF-1/54-DCN lost its capacity in CXCR4 binding. Therefore we examined whether SDF-1/54-DCN had an inhibitory effect on SDF- $1 \alpha$ chemotaxis. When induced by SDF- $1 \alpha$, the cells pretreated by SDF-1/54-DCN showed low chemotaxis, while untreated cells maintained high chemotaxis (Fig. 4C). This inhibitory effect became dose-dependent at a concentration range of $100-500 \mathrm{~nm}$, from which we presumed that SDF-1/54-DCN probably preserved capacity for binding to CXCR4. Moreover, the receptor internalization was investigated to determine whether SDF-1/54-DCN was able to bind to CXCR4 on MOLT-4 cells since CXCR4 internalization is dependent on its ligand binding. ${ }^{27)}$ MOLT-4 cells were incubated with SDF-1/54-DCN (500 nM) for $2 \mathrm{~h}$, and the presence of CXCR4 on the cell surface was observed by flow cytometry. A reduced level of CXCR4 on the surface of MOLT-4 cells was detected (mean fluorescence density decreased from 71.5 to $37 \%$ ) (Fig. 4D). In addition, the cell viability was more than $95 \%$ under the above experimental conditions. In a word, above evidences confirmed that the 
A

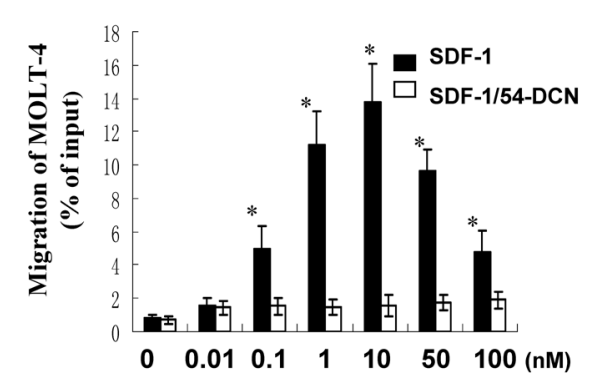

C

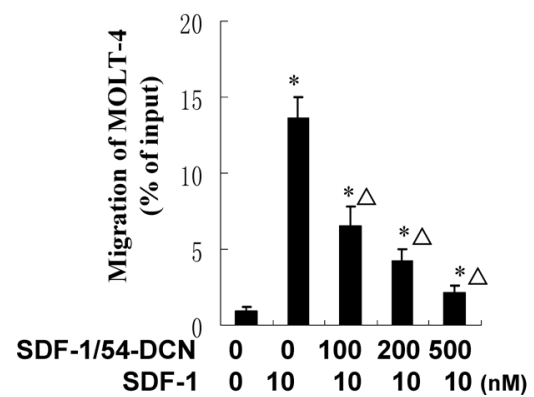

B

SDF-1 SDF-1/54-DCN

$\begin{array}{llllll}5 & 50 & 20 & 10 & 5 & (\mathrm{nM})\end{array}$

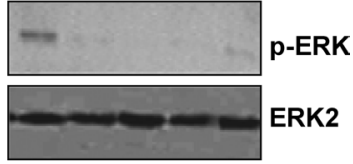

D

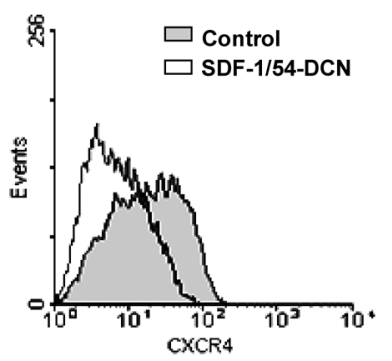

Fig. 4. Inhibitory Effect of SDF-1/54-DCN on SDF- $1 \alpha$-Mediated Chemotaxis in MOLT-4 Cells

(A) Chemotactic activities of both SDF- $1 \alpha$ and SDF-1/54-DCN in the MOLT- 4 cell line were observed using Transwell trays with the membrane pore of $5 \mu \mathrm{m}$. The control was the migrating cells without any treatment. The results are presented as mean \pm standard deviation from 3 separate experiments conducted in triplicate for each condition. $* p<0.05$ $v s$. SDF-1 $\alpha$. (B) Failure of SDF-1/54-DCN to trigger ERK signaling. MOLT- 4 cells were stimulated with SDF-1 $\alpha$ or SDF-1/54-DCN for 2 min. Cells were subjected to immunoblotting analysis with a mouse anti-phospho-ERK antibody and a rabbit anti-ERK2 antibody. Images are the representative gels of at least 3 experiments. (C) SDF-1/54-DCN suppressed the chemotactic ability of SDF- $1 \alpha$. The results are presented as mean \pm standard deviation from 3 separate experiments conducted in triplicate for each condition. $* p<0.05 v s$. control; $\triangle p<0.05 v s$. SDF-1 $\alpha$ alone (positive control). (D) Ability of SDF-1/54-DCN to induce CXCR4 internalization. MOLT-4 cells were treated with SDF-1/54$\mathrm{DCN}$ for $2 \mathrm{~h}$. CXCR4 on the cell surface was stained with PE-conjugated anti-human CXCR4 antibody and observed by flow cytometry. Data shown are typical representatives of three independent experiments.
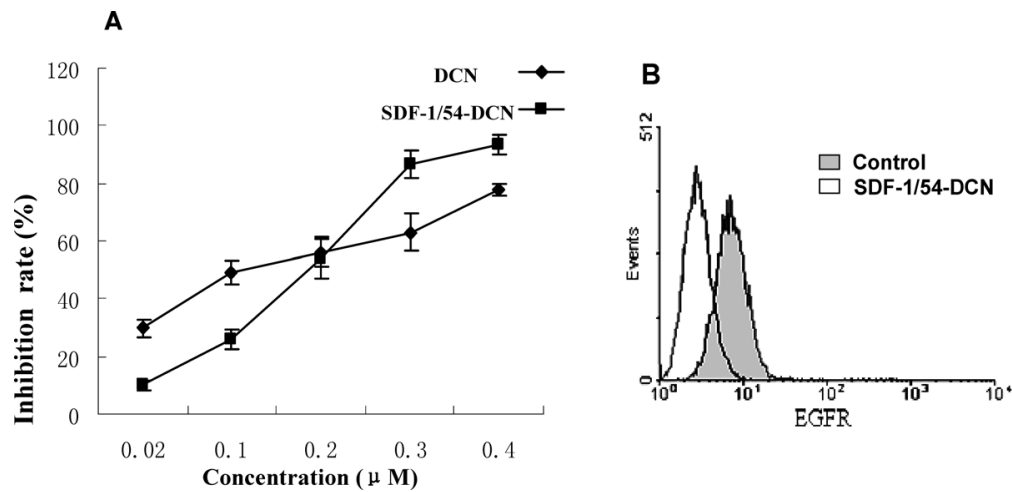

Fig. 5. Inhibitory Effect of SDF-1/54-DCN on Proliferation in A431 Cells

(A) Inhibitory effect of SDF-1/54-DCN on A431 cell proliferation was assessed by MTT assay. The results are presented as mean \pm standard deviation from 3 separate experiments conducted in triplicate for each condition. (B) Ability of SDF-1/54-DCN to induce EGFR internalization. A431 cells were treated with SDF-1/54-DCN for $2 \mathrm{~h}$. EGFR on the cell surface was stained with FITC-conjugated anti-human EGFR antibody and observed by flow cytometry. Data shown are typical representatives of three independent experiments.

binding ability of SDF-1/54-DCN to CXCR4 was not affected due to the fusion of DCN, and SDF-1/54-DCN inhibited chemotactic ability of SDF- $1 \alpha$ by competitively blocking CXCR4, manifesting that this novel recombinant protein might be a potential effective antagonist of CXCR4.

SDF-1/54-DCN Inhibited Proliferation in A431 Cells DCN was reported to potentially inhibit cell proliferation, and this inhibitory effect might be attributed to the ability of DCN to induce the internalization and degradation of EGFR. ${ }^{17)}$ The inhibitory effect of SDF-1/54-DCN on the proliferation of A431 cells overexpressing EGFR was analyzed by the MTT assay. The results showed that SDF-1/54-DCN could suppress the proliferation of A431 cells in a dose-dependent manner, and this inhibitory effect appeared to be more significant $(p<0.01)$ at a concentration range of $0.2-$ $0.4 \mu \mathrm{M}$ (Fig. 5A) (The percentage of cell viability between treated and untreated groups had no significant difference $p<0.05)$. To investigate whether the downregulation of EGFR is involved in the inhibitory effect of SDF-1/54-DCN on cell proliferation, A431 cells were incubated with SDF1/54-DCN $(1 \mu \mathrm{M})$ for $2 \mathrm{~h}$, and the presence of EGFR was then observed on the cell surface by flow cytometry with an 


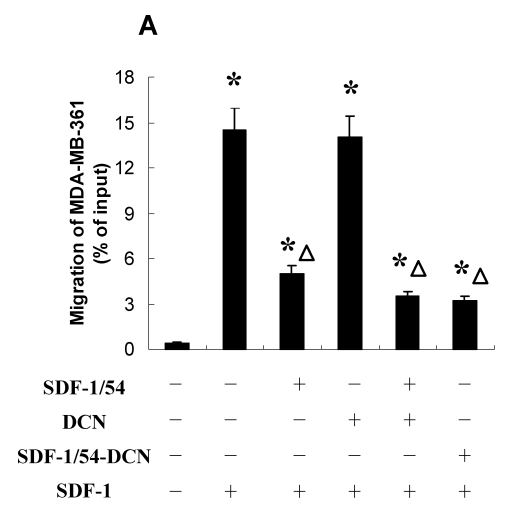

B

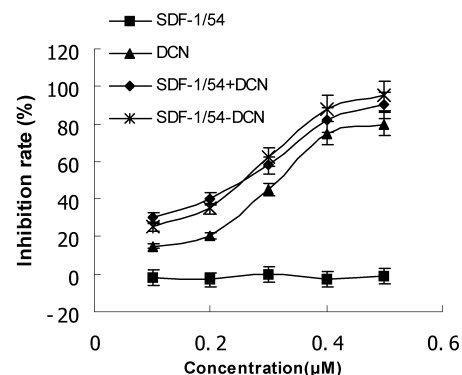

Fig. 6. Inhibitory Effect of SDF-1/54-DCN on SDF-1 $\alpha$-Mediated Chemotaxis and Proliferation in MDA-MB-361 Cells

(A) Inhibitory effect of SDF-1/54-DCN on the chemotaxis induced by SDF-1 $\alpha$ in the MDA-MB-361 cell. The cell migration activated by SDF- $1 \alpha(10 \mathrm{nM})$ with pretreatments of either SDF-1/54 (500 nM), DCN (500 nM) alone or combined both, SDF-1/54-DCN (500 nM) was examined. The control was the migrating cells without any treatment. The results are presented as mean \pm standard deviation from 3 separate experiments conducted in triplicate for each condition. $* p<0.05 v s$. control; $\Delta p<0.05 v s$. SDF- $1 \alpha$ alone (positive control). (B) Inhibitory effect of SDF-1/54-DCN on MDA-MB-361 cell proliferation assessed by MTT assay. The results are presented as mean \pm standard deviation from 3 separate experiments conducted in triplicate for each condition.

anti-EGFR antibody. The EGFR level was found to have decreased from 40.5 to $7.7 \%$ on the A431 cell surface (Fig. 5B) after exposure to SDF-1/54-DCN. These data indicated that the DCN domain, as a ligand for EGFR in this new molecule, contributed to the inhibition of cell proliferation by blocking the EGFR.

SDF-1/54-DCN Inhibited Not Only SDF-1 $\alpha$-Mediated Chemotaxis But Also Proliferation in MDA-MB-361 Cells In order to compare the bioactivity of SDF-1/54-DCN with SDF-1/54, DCN alone or the combined on inhibiting tumor cells chemotaxis as well as proliferation, MDA-MB-361 cells were used as they overexpressed both CXCR4 and EGFR. ${ }^{28,29)}$ The results from chemotaxis inhibition assay showed that SDF- $1 \alpha$-mediated cell migration was blocked when pretreated with $500 \mu \mathrm{M}$ of SDF-1/54, SDF-1/54-DCN or SDF-1/54 and DCN combination respectively. And the blocking effect produced by SDF-1/54-DCN or SDF-1/54 and DCN combination was more remarkable as compared to SDF-1/54 $(p<0.05)$. But there were no significant differences between the two former groups $(p>0.05)$. On the other hand, we observed that all of DCN, SDF-1/54-DCN as well as SDF-1/54 and DCN combination could inhibit the proliferation of MDA-MB-361 cells in a concentration dependent manner $(0.1-0.5 \mu \mathrm{M})$. The inhibitory activity of either SDF$1 / 54-\mathrm{DCN}$ or SDF-1/54 and DCN combination was higher than DCN alone at a concentration range of $0.1-0.3 \mu \mathrm{M}$ $(p<0.05)$, and there were no obvious differences between the two former groups $(0.1-0.5 \mu \mathrm{M}, p>0.05)$. Under the above conditions, the cytotoxicity of SDF-1/54-DCN did not observed. These data indicated that the two functional domains in SDF-1/54-DCN structure were able to exert their own bioactivity, such as blocking chemotactic ability of SDF- $1 \alpha$ or inhibiting proliferation.

\section{CONCLUSIONS}

In order to achieve the expected dual effects of SDF-1/54DCN, several critical problems should be considered and resolved. The first issue is how to maintain the bioactivity of both SDF-1/54 and DCN in the chimera. It is known that the hinge region of human IgG1 heavy chain is a short peptide rich in proline, presents high flexibility, and is a perfect conjunctive fragment. ${ }^{30)}$ Based on the features of the hinge region, we used the gene segment of the human IgG1 heavy chain as a linker between SDF-1/54 and DCN to maintain the spatial structural integrity of the chimera protein. The second is how to arrange the sequences of SDF-1/54 and DCN domains. The N-terminal loop of SDF- $1 \alpha$ (amino acids 12 17) has been demonstrated as a key site for its original binding to CXCR4, while the sixth leucine-rich repeat in the center (III section) of the core protein is the binding site of DCN to EGFR. Therefore, the optimal arrangement of the recombinant gene is that DCN located in the C-terminus of SDF$1 / 54$ with the hinge region of human IgG1 in between, thereby preventing the structural hindrance of the $\mathrm{N}$-terminus of SDF-1/54. The evidences from in vitro experiments confirmed that the hinge region can be used as a linker between SDF-1/54 and DCN to maintain the natural structure of both the components and block the two signal pathways mediated by CXCR 4 and EGFR.

Whether the two functional domains of SDF-1/54-DCN effectively target both CXCR4 and EGFR respectively is the key to improve the targeting capability of SDF-1/54 to the tumor cells that express both CXCR4 and EGFR on their surface in vivo. Our results revealed that SDF-1/54-DCN retained its affinity to CXCR4 while abrogating the internal activity of CXCR4. Compared with SDF- $1 \alpha$, SDF-1/54-DCN in MOLT- 4 cells exhibited a significantly decreased chemotactic ability, and the cells pretreated with SDF-1/54-DCN were unreactive to the chemotaxis of SDF- $1 \alpha$. Although SDF-1/54-DCN induced internalization of CXCR4 on the surface of MOLT- 4 cells, the capacity of SDF-1/54-DCN induced phosphorylation of ERK was obviously weak. Also, SDF-1/54-DCN strongly inhibited SDF- $1 \alpha$-mediated chemotaxis in MDA-MB-361 cells, but did not in HEK293 cells without expression of both CXCR4 and EGFR (data not shown). These evidences indicated that SDF-1/54-DCN remained the antagonistic capacity of SDF-1/54 to CXCR4. Additionally, SDF-1/54-DCN could significantly inhibit the proliferation of the A431 cell line overexpressing EGFR in a dose-dependent manner, and induce the internalization and degradation of EGFR. The same proliferation inhibition in- 
duced by SDF-1/54-DCN was also proved in MDA-MB-361 cells, but not in HEK293 cells. Since one of important functions of EGFR is to promote cell proliferation, these evidences hint that this new recombinant chimera possesses an effective DCN bioactivity.

Recently, some studies have noticed a few clues that indicated the occurrence of "cross-talk" events between CXCR4 and EGFR intracellular pathways that might link different proliferation and migration pathways of tumors. For instance, EGF could induce the expression of CXCR4 in non-small cell lung cancer cells, ${ }^{31)}$ while SDF- $\alpha$ could stimulate the growth of ovarian cancer cells through EGF receptor transactivation. ${ }^{32}$ ) These findings implied that compared with the inhibition of either EGFR or CXCR4 signaling, simultaneous blocking of these two receptors may be a more effective strategy for the prevention and treatment of cancer. Our data from MDA-MB-361 cells were apparently consistent with above reports. The inhibitory effects of SDF-1/54-DCN on SDF- $1 \alpha$-mediated chemotaxis and proliferation of MDAMB-361 cells were enhanced as compared to SDF-1/54 or DCN alone, which may be attributed to "cross-talk" events between CXCR4 and EGFR intracellular pathways.

In a word, in this study we had developed a novel recombinant chimera SDF-1/54-DCN and demonstrated in vitro that it had dual inhibitory effects on proliferation and chemotaxis of tumor cells since maintained the bioactivities of both SDF-1/54 and DCN. Additionally, experiments in vivo demonstrated that DCN could suppress tumorigenicity by specifically targeting EGFR-overexpressing tumor cells. ${ }^{18}$ These evidence indicated that our strategy was promising to increase the targeting ability of SDF-1/54 on the tumor cells coexpressing CXCR4 and EGFR. However, targeting ability of SDF-1/54-DCN to relative solid tumors and pharmacokinetics character in vivo should be further confirmed in animal experiments.

Acknowledgements This project is supported partly by grants from the National and Provincial Natural Science Foundation of China (No. 30572209 \& No. 04010459).

\section{REFERENCES}

1) Arya M., Patel H. R., McGurk C., Tatoud R., Klocke H., Masters J., Williamson M., J. Exp. Ther. Oncol., 4, 291-303 (2004).

2) Darash-Yahana M., Pikarsky E., Abramovitch R., Zeira E., Pal B., Karplus R., Beider K., Avniel S., Kasem S., Galun E., Peled A., FASEB J., 18, 1240-1242 (2004).

3) Liang Z., Yoon Y., Votaw J., Goodman M. M., Williams L., Shim H., Cancer Res., 65, 967-971 (2005).

4) Muller A., Homey B., Soto H., Ge N., Catron D., Buchanan M. E., McClanahan T., Murphy E., Yuan W., Wagner S. N., Barrera J. L., Mohar A., Verastegui E., Zlotnik A., Nature (London), 410, 50-56 (2001).
5) Uchida D., Onoue T., Tomizuka Y., Begum N. M., Miwa Y., Yoshida H., Sato M., Mol. Cancer Res., 5, 685-694 (2007).

6) Kijima T., Maulik G., Ma P. C., Tibaldi E. V., Turner R. E., Rollins B., Sattler M., Johnson B. E., Salgia R., Cancer Res., 62, 6304-6311 (2002).

7) Taichman R. S., Cooper C., Keller E. T., Pienta K. J., Taichman N. S., McCauley L. K., Cancer Res., 62, 1832-1837 (2002).

8) Cai S. H., Tan Y., Ren X. D., Li X. H., Cai S. X., Du J., Acta Pharmacol. Sin., 25, 152-160 (2004).

9) Tan Y., Du J., Cai S., Li X., Ma W., Guo Z., Chen H., Huang Z., Xiao J., Cai L., Cai S., Exp. Hematol., 34, 1553-1562 (2006).

10) Horuk R., Nature (London), 393, 524-525 (1998)

11) Olayioye M. A., Neve R. M., Lane H. A., Hynes N. E., EMBO J., 19 3159-3167 (2000).

12) Chen P., Cameron R., Wang J., Vallis K. A., Reilly R. M., J. Nucl. Med., 44, 1469-1478 (2003).

13) Leygue E., Snell L., Dotzlaw H., Troup S., Hiller-Hitchcock T., Murphy L. C., Roughley P. J., Watson P. H., J. Pathol., 192, 313-320 (2000).

14) Reed C. C., Waterhouse A., Kirby S., Kay P., Owens R. T., McQuillan D. J., Iozzo R. V., Oncogene, 24, 1104-1110 (2005).

15) Troup S., Njue C., Kliewer E. V., Parisien M., Roskelley C., Chakravarti S., Roughley P. J., Murphy L. C., Watson P. H., Clin. Cancer Res., 9, 207-214 (2003).

16) Santra M., Reed C. C., Iozzo R. V., J. Biol. Chem., 277, 35671-35681 (2002).

17) Zhu J. X., Goldoni S., Bix G., Owens R. T., McQuillan D. J., Reed C. C., Iozzo R. V., J. Biol. Chem., 280, 32468-32479 (2005).

18) Seidler D. G., Goldoni S., Agnew C., Cardi C., Thakur M. L., Owens R. T., McQuillan D. J., Iozzo R. V., J. Biol. Chem., 281, 26408-26418 (2006).

19) Azemar M., Schmidt M., Arlt F., Kennel P., Brandt B., Papadimitriou A., Groner B., Wels W., Int. J. Cancer, 86, 269-275 (2000).

20) Xu Y. M., Wang L. F., Jia L. T., Qiu X. C., Zhao J., Yu C. J., Zhang R., Zhu F., Wang C. J., Jin B. Q., Chen S. Y., Yang A. G., J. Immunol., 173, 61-67 (2004).

21) Jain P. T., Pento J. T., Graves D. C., J. Pharmacol. Toxicol. Methods, 27, 203-207 (1992).

22) Mosmann T., J. Immunol. Methods, 65, 55-63 (1983).

23) Markossian K. A., Kurganov B. I., Biochemistry (Mosc.), 69, 971984 (2004).

24) Li M., Su Z. G., Janson J. C., Protein Expr. Purif., 33, 1-10 (2004).

25) Bleul C. C., Fuhlbrigge R. C., Casasnovas J. M., Aiuti A., Springer T. A., J. Exp. Med., 184, 1101-1109 (1996).

26) Zhao M., Discipio R. G., Wimmer A. G., Schraufstatter I. U., Mol. Pharmacol., 69, 66-75 (2006)

27) Hesselgesser J., Liang M., Hoxie J., Greenberg M., Brass L. F., Orsini M. J., Taub D., Horuk R., J. Immunol., 160, 877-883 (1998).

28) Cabioglu N., Summy J., Miller C., Parikh N. U., Sahin A. A., Tuzlali S., Pumiglia K., Gallick G. E., Price J. E., Cancer Res., 65, 64936497 (2005).

29) Normanno N., De Luca A., Maiello M. R., Campiglio M., Napolitano M., Mancino M., Carotenuto A., Viglietto G., Menard S., J. Cell Physiol., 207, 420- 427 (2006).

30) Saphire E. O., Stanfield R. L., Crispin M. D., Parren P. W., Rudd P. M., Dwek R. A., Burton D. R., Wilson I. A., J. Mol. Biol., 319, 9-18 (2002).

31) Phillips R. J., Mestas J., Gharaee-Kermani M., Burdick M. D., Sica A., Belperio J. A., Keane, M. P., Strieter R. M., J. Biol. Chem., 280, 22473-22481 (2005).

32) Porcile C., Bajetto A., Barbieri F., Barbero S., Bonavia R., Biglieri M., Pirani P., Florio T., Schettini G., Exp. Cell Res., 308, 241—253 (2005). 